\title{
Mechanical Properties of MWCNT/Epoxy Nanocomposite Films
}

\section{Shiuh-Chuan Her* and Ting-Yu Shiu}

Department of Mechanical Engineering, Yuan Ze University, Chung.Li, Taiwan

\section{Introduction}

Since the discovery of carbon nanotubes (CNTs) by Iijima [1] in 1991, CNTs have attracted considerable attention in recent years. The excellent mechanical, thermal and electrical properties of CNTs may hold the promise as a potential reinforcing material in polymer matrix composite. The improvements in stiffness and strength because of the addition of CNTs in polymer matrix have been reported [2-4]. Most studies on CNT reinforced nanocomposites have focused on their marco behavior of the material in bulk state. Even though these studies are quite useful in establishing the properties of the nanocomposites. Use of CNTs in thin films may find potential applications in micro devices, electronic packaging and field emission display. Only a few researchers have studied the mechanical properties of CNTs reinforced composite film [5]. For the implementation of CNTs reinforced film in engineering applications, accurate prediction of the film properties is essential. In this work, the multi-walled carbon nanotubes (MWCNT) reinforced epoxy thin films were prepared by a hot-pressing process. The uniaxial tensile tests were conducted to evaluate the mechanical properties of MWCNT reinforced composite films. The effects of MWCNT content on the mechanical properties of the MWCNT/epoxy composite were investigated.

\section{Film Preparation}

The multi-walled carbon nanotubes produced via chemical vapor deposition method were purchased from Shenzhen Nanotechnologies Port Co. and purified to $>95 \%$. The MWCNTs had diameter ranging from $40 \mathrm{~nm}$ to $60 \mathrm{~nm}$, and the length ranging from $5 \mu \mathrm{m}$ to $15 \mu \mathrm{m}$. The matrix used was part A: epoxy 6620 and part B: hardener AH150, both purchased from Glad Co. Taiwan. To prepare MWCNT/epoxy composite, MWCNT powder was directly added into a liquid epoxy, and the solution was sonicated for five hours to separate the aggregation of the MWCNTs and achieve good dispersion. Then, the epoxy hardener H320 was mixed into the MWCNT/epoxy solution, and softly stirred it for about 5 minutes. After that the solution was placed in a vacuum chamber for about 30 minutes to remove the bubbles induced from the stirring. Finally, the nanocomposite suspension was poured onto a mold as shown in Figure 1. The mold was supported and covered by steel plates and placed on the hot-press machine at temperature of $50^{\circ} \mathrm{C}$ and under the pressure of $100 \mathrm{~N} / \mathrm{cm}^{2}$. The thickness of the film on the substrate was controlled by a spacer. Prior to pouring the nanocomposite solution, the substrate was cleaned in soap solution,

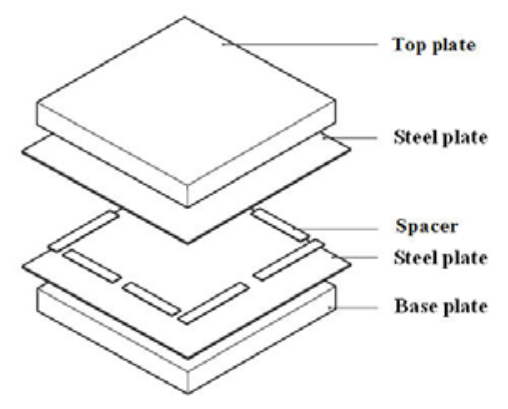

Figure 1: Hot pressing mold. submerged in acetone solution for 10 minutes after rising with distilled water. Then the substrate was dried with hot air before the application of pouring. A nanocomposite film with thickness of $200 \mu \mathrm{m}$ was obtained after 3 hours of curing in the hot-pressing mold. Two different contents of MWCNT ( $1 \mathrm{wt} \%$ and $2 \mathrm{wt} \%$ ) in the nanocomposite films were prepared. Samples without the MWCNT addition were also fabricated for comparison.

\section{Uniaxial Tensile Test}

Uniaxial tensile tests were conducted using a Hounsfield material test system according to the ASTM D882 test procedure. The asprepared nanocomposite films were cut in $185 \mathrm{~mm} \times 15 \mathrm{~mm} \times 0.23$ $\mathrm{mm}$ to form a tensile test specimen as shown in Figure 2. The loading rate was $5 \mathrm{~mm} / \mathrm{min}$. Three tests were performed for each sample. The average of three measured values was taken for each sample, and the measurements were reported as the mean value. Figure 3 shows three typical stress-strain curves of nanocomposites with $0 \mathrm{wt} \%, 1 \mathrm{wt} \%$ and 2 wt $\%$ MWCNTs, respectively. The Young's modulus was calculated from the slope in the linear region of the stress-strain curve. The numerical values of Young's modulus, yield strength, tensile strength and fracture strain are summarized in Table 1. It can be seen that, in comparison with neat epoxy, the MWCNT/epoxy nanocomposite film exhibits higher Young's modulus, yield strength, tensile strength and fracture strain. As listed in Table 1, the Young's modulus increases from $1.15 \mathrm{GPa}$ for neat epoxy to $1.35 \mathrm{GPa}$ and $1.52 \mathrm{GPa}$ for MWCNT/epoxy nanocomposites with $1 \mathrm{wt} \%$ and $2 \mathrm{wt} \%$ of MWCNTs, respectively. The tensile strength increases from $31.51 \mathrm{GPa}$ for neat epoxy to 35.65 $\mathrm{GPa}$ and $42.80 \mathrm{GPa}$ for MWCNT/epoxy nanocomposites with $1 \mathrm{wt} \%$ and $2 \mathrm{wt} \%$ of MWCNTs, respectively. At the same time, the strain at break increases from 3.9\% for neat epoxy to $4.3 \%$ and $5.0 \%$ for MWCNT/epoxy nanocomposites with $1 \mathrm{wt} \%$ and $2 \mathrm{wt} \%$ of MWCNTs, respectively.

The MWCNTs/epoxy nanocomposite films were successfully prepared by a hot pressing process. The mechanical properties of the

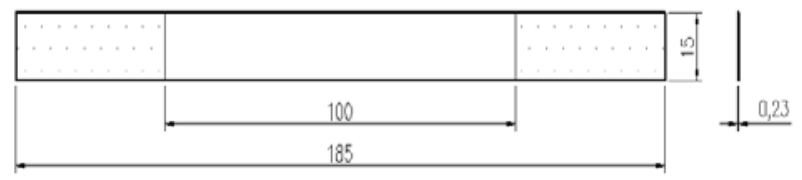

Figure 2: Tensile test specimen (unit: $\mathrm{mm}$ ).

*Corresponding author: Shiuh-Chuan Her, Department of Mechanical Engineering, Yuan Ze University, Chung.Li, Taiwan, Tel: 8863-4638800; E-mail: mesch@saturn.yzu.edu.tw

Received February 19, 2014; Accepted February 19, 2014; Published March 03, 2014

Citation: Her SC, Shiu TY (2014) Mechanical Properties of MWCNT/Epoxy Nanocomposite Films. J Appl Mech Eng 3: e128. doi:10.4172/2168-9873.1000e128

Copyright: ( $) 2014 \mathrm{Her}$ SC, et al. This is an open-access article distributed under the terms of the Creative Commons Attribution License, which permits unrestricted use, distribution, and reproduction in any medium, provided the original author and source are credited. 
Citation: Her SC, Shiu TY (2014) Mechanical Properties of MWCNT/Epoxy Nanocomposite Films. J Appl Mech Eng 3: e128. doi:10.4172/2168$9873.1000 \mathrm{e} 128$

Page 2 of 2

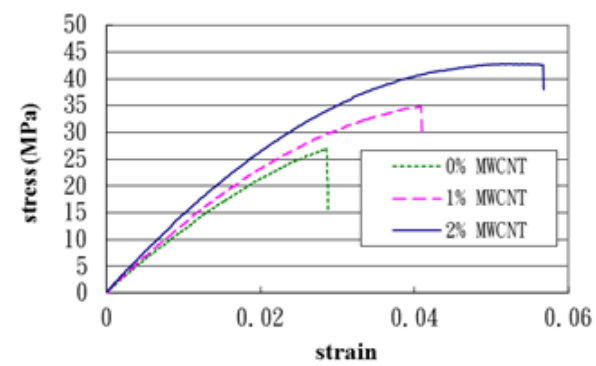

Figure 3: Stress-strain curves of nanocomposite films with various MWCNT wt\%.

\begin{tabular}{|l|l|l|l|}
\hline $\begin{array}{l}\text { MWCNT wt \% material } \\
\text { properties }\end{array}$ & $0 \%$ & $1 \%$ & $2 \%$ \\
\hline Young's modulus (GPa) & 1.15 & 1.35 & 1.52 \\
\hline Yield strength (MPa) & 23.07 & 25.97 & 27.50 \\
\hline Tensile strength (MPa) & 31.51 & 35.65 & 42.80 \\
\hline Fracture strain \% & 3.9 & 4.3 & 5.0 \\
\hline
\end{tabular}

Table 1: Mechanical Properties of Nanocomposite Films with various Wt \% of MWCNT
MWCNTs reinforced epoxy films were measured by uniaxial tensile test. Experimental results show that the addition of the MWCNTs into epoxy matrix exhibits significantly improvement in the Young's modulus, tensile strength, and yield strength of the nanocomposite film compared with pure epoxy.

\section{References}

1. lijimaS (1991) Helicalmicrotubes of graphitic carbon. Nature 354: 56-58.

2. Cho J, Daniel IM (2008) Reinforcement of carbon/epoxy composites with multi-wall carbon nanotubes and dispersion enhancing block copolymers. ScriptaMaterialia 58: 533-536.

3. Yu N, Zhang ZH, He SY (2008) Fracture toughness and fatigue life of MWCNT/ epoxy composites. Materials Science and Engineering: A 494: 380-384.

4. Ci L, Bai J (2006) The reinforcement role of carbon nanotubes in epoxy composites with different matrix stiffness. Composites Science and Technology 66: 599-603.

5. Wu DC, Shen L, Low JE, Wong SY, Li X, et al. (2010) Multi-walled carbon nanotube/polyimide composite film fabricated through electrophoretic deposition. Polymer 51: 2155-2160. 